\title{
Adaptive Neuro-Fuzzy Inference System for Classification of Urodynamic Test
}

\author{
Mohanad A.Deaf, Mohamed A.A.Eldosoky \\ Dept. of Biomedical Engineering, Faculty of Engineering, \\ Helwan University, Egypt
}

\begin{abstract}
Urodynamic test is a method that assesses how the bladder and urethra are performing their functions of storing and releasing of the urine. The aim of this paper is to develop an approach for classifying of Urodynamic result to normal and abnormal value using adaptive neuro fuzzy inference system. The data of Urodynamic were obtained from the Institute of Experimental Clinical Research. Eight Urodynamic Components are used as input for the prediction model. Simulations were run in Matlab. The simulation results demonstrate that the model successfully to classify the Urodynamic Components to normal and abnormal values with an accuracy rate of $97.30 \%$.
\end{abstract}

Keywords: Adaptive neuro-fuzzy inference system (ANFIS), ANFIS Classifier, Urodynamic testing (UDS).

\section{INTRODUCTION}

Urodynamic testing or Urodynamics is a study that assesses how the bladder and urethra are performing their functions of storing and releasing urine [1]. Therefore, urodynamic tests can help explain symptoms such as: incontinence, frequent urination, problems associated with emptying the bladder.

The term Urodynamics was first used by Davis in 1954 [2] as the study of the storage and evacuation by the bladder UDS evaluate bladder function (capacity, sensation, accommodation, and contractility) [3] to confirm a physician's differential diagnosis. Urodynamic tests include uroflowmetry, post void residual measurement, cystometric test, leak point pressure measurement, pressure flow study and electromyography[4].

Uroflowmetry is the measurement of urine speed and volume .Post void residual measures the amount of urine left in the bladder after urination. A cystometric test measures how much urine the bladder can hold, how much pressure builds up inside the bladder as it stores urine, and how full it is when the urge to urinate begins. leak point pressure measures pressure at the point of leakage during a cystometric test.

A pressure flow study measures the bladder pressure required to urinate and the flow rate a given pressure generates. Electromyography uses special sensors to measure the electrical activity of the muscles and nerves in and around the bladder and the sphincters.

In this study we had applied the parameters that were taken from practical urodynamics according to Institute of Experimental Clinical Research [5] [6] [7], which are : maximum detrusor pressure, opening pressure, detrusor pressure at maximum flow rate, volume of urine voided/micturition , maximum urine flow rate, maximum bladder contractility, micturition frequency and number of micturitions. The physician decides if urodynamics result is normal or not by minimum and maximum rang value for each component .

The aim of this study is developed to a new approach based on adaptive neuro fuzzy inference system (ANFIS) for rapid decide on the urodynamic test as normal or not with a high accuracy rate. Adaptive neuro-fuzzy inference system was presented for classification of the urodynamic testing.

Adaptive network-based fuzzy inference system (ANFIS) is a kind of artificial neural network that is based on TakagiSugeno fuzzy inference system. Adaptive Neuro-Fuzzy Inference System (ANFIS) is one of the most successful schemes which combine the benefits of these two powerful paradigms into a single capsule [8] [9]. An ANFIS works by applying neural learning rules to identify and tune the parameters and structure of a Fuzzy Inference System (FIS). There are several features of the ANFIS which enable it to achieve great success in a wide range of scientific applications. The attractive features of an ANFIS include: easy to implement, fast and accurate learning, strong generalization abilities, excellent explanation facilities through fuzzy rules, and easy to incorporate both linguistic and numeric knowledge for problem solving [10] [11] [12].

This paper is organized as follows: The theoretical background of urodynamic testing is demonstrated in the introduction section. Section 2 presents review about the adaptive neuro fuzzy inference scheme used in the design of the classifier. The structural information of the dataset is presented in section 3. System validation and discussions on it, is given in section 4 . This is followed by the conclusions in the concluding section, and references.

\section{ADAPTIVE-NEURO-FUZZY INFERENCE SYSTEM (ANFIS) OVERVIEW}

The technique was developed in the early 1990s. According to the neuro-fuzzy approach, a neural network is proposed to implement the fuzzy system, so that the structure and parameter identification of the fuzzy rule base are accomplished by defining, adapting and optimizing the topology and the parameters of the corresponding neuro-fuzzy network, based only on the available data. The network can be regarded both as an adaptive fuzzy inference system with the capability of learning fuzzy rules from data, and as a connectionist architecture provided with linguistic meaning. 


\subsection{The ANFIS Architecture}

A typical architecture of an ANFIS, in which a circle indicates a fixed node, whereas a square indicates an adaptive node, is shown in Fig. 1.

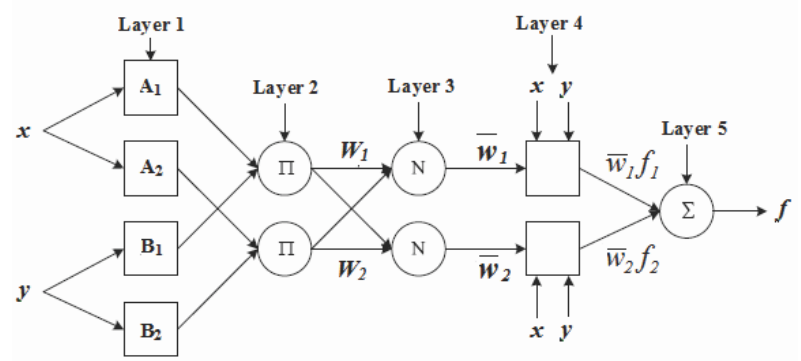

Fig. 1: ANFIS architecture

In this connectionist structure, there are input and output nodes, and in the hidden layers, there are nodes functioning as membership functions (MFs) and rules. This eliminates the disadvantage of a normal feed forward multilayer network, which is difficult for an observer to understand or to modify. For simplicity, we assume that the examined FIS has two inputs and one output. For a first-order Sugeno fuzzy model, a typical rule set with two fuzzy "if- then" rules can be expressed as follows:

Rule 1: If $x$ is $\mathrm{A} 1$ and $\mathrm{y}$ is $\mathrm{B} 1$, then $\mathrm{fl}_{1}=p_{1} x+\mathrm{q} 1 \mathrm{y}+\mathrm{r} 1$

Rule 2: If $x$ is $\mathrm{A}_{2}$ and $\mathrm{y}$ is $\mathrm{B}_{2}$, then $f_{2}=p_{1} x+\mathrm{q} 2 \mathrm{y}+\mathrm{r} 2$

Where $\mathrm{x}$ and $\mathrm{y}$ are the two crisp inputs, and $\mathrm{Ai}$ and $\mathrm{Bi}$ are the linguistic labels associated with the node function.

As indicated in Fig. 3, the system has a total of five layers. The functioning of each layer is described as follows [13] [14].

Input node (Layer 1): Nodes in this layer contains membership functions. Parameters in this layer are referred to as premise parameters. Every node $\mathrm{i}$ in this layer is a square and adaptive node with a node function:

$O_{2, i}=w_{i}=\mu_{A_{i}}(x) \quad$ For $i=1,2$

Where $\mathrm{x}$ is the input to node $\mathrm{i}$, and $\mathrm{A}_{\mathrm{i}}$ is the linguistic label (small, large, etc.) associated with this node function. In other words, $O_{2, i}$ is the membership function of $\mathrm{A}_{\mathrm{i}}$ and it specifies the degree to which the given $\mathrm{x}$ satisfies the quantifier $\mathrm{A}_{\mathrm{i}}$.

Rule nodes (Layer 2): Every node in this layer is a circle node labeled II, whose output represents a firing strength of a rule. This layer chooses the minimum value of two input weights. In this layer, the AND/OR operator is applied to get one output that represents the results of the antecedent for a fuzzy rule, that is, firing strength. It means the degrees by which the antecedent part of the rule is satisfied and it indicates the shape of the output function for that rule. The node generates the output (firing strength) by cross multiplying all the incoming signals:

$$
O_{2, i}=w_{i}=\mu_{A_{i}}(x) \quad \mu_{B_{i}}(y) \quad i=1,2
$$

Average nodes (Layer 3): Every node in this layer is a circle node labeled $\mathrm{N}$. The $\mathrm{i}^{\text {th }}$ node calculates the ratio between the $i^{\text {th }}$ rule's firing strength to the sum of all rules' firing strengths. Every node of these layers calculates the weight, which is normalized. For convenience, the outputs of this layer are called normalized firing strengths.

$$
O_{3, i}=\overline{w_{l}}=\frac{w_{i}}{w_{1}+w_{2}} \quad i=1,2
$$

Consequent nodes (Layer 4): This layer includes linear functions, which are functions of the input signals. This means that the contribution of $i^{\text {th }}$ rule's towards the total output or the model output and/or the function defined is calculated. Every node $\mathrm{i}$ in this layer is a square node with a node function:

$$
O_{4, i}=\overline{w_{l}} f_{i}=\overline{w_{l}}\left(p_{i} x+q_{i} y+r_{i}\right)
$$

Where $\overline{w_{l}}$ is the output of layer 3 , and $\{\mathrm{pi}, \mathrm{qi}, \mathrm{ri}\}$ is the parameter set of this node. These parameters are referred to as consequent parameters

Output node (Layer 5): The single node in this layer is a fixed node labeled $\sum$, which computes the overall output by summing all incoming signals:

$$
O_{5, i}=\sum_{i} \overline{w_{l}} f_{i}=\sum_{i} \frac{w_{i} f_{i}}{w_{i}}
$$

\subsection{ANFIS Learning process (Hybrid- learning Algorithm)}

As mentioned earlier, both the premise (non-linear) and consequent (linear) parameters of the ANFIS should be tuned, utilizing the so-called learning process, to optimally represent the factual mathematical relationship between the input space and output space. Normally, as a first step, an approximate fuzzy model is initiated by the system and then improved through an iterative adaptive learning process. Basically, ANFIS takes the initial fuzzy model and tunes it by means of a hybrid technique combining gradient descent back propagation and mean least-squares optimization algorithms. At each epoch, an error measure, usually defined as the sum of the squared difference between actual and desired output, is reduced. Training stops when either the predefined epoch number or error rate is obtained.

There are two passes in the hybrid learning procedure for ANFIS. In the forward pass of the hybrid learning algorithm, functional signals go forward till layer 4 and the consequent parameters are identified by the least squares estimate. In the backward pass, the error rates propagate backward and the premise parameters are updated by the gradient descent. When the values of the premise parameters are learned, the overall output (f) can be expressed as a linear combination of the consequent parameters [15] [16] [17]:

$$
\begin{gathered}
f=\frac{w_{1}}{w_{1}+w_{2}} f_{1}+\frac{w_{2}}{w_{1}+w_{2}} f_{2} \\
=\overline{w_{1}} f_{1}+\overline{w_{2}} f_{2} \\
=\left(\overline{w_{1}} x\right) p_{1}+\left(\overline{w_{1}} y\right) q_{1}+\left(\overline{w_{1}}\right) r_{1}+\left(\overline{w_{2}} x\right) p_{2}+\left(\overline{w_{2}} y\right) q_{2} \\
+\left(\overline{w_{2}}\right) r_{2}
\end{gathered}
$$




\section{DEVELOPMENT OF ANFIS CLASSIFIER}

\subsection{Data preparation (Urodynamic parameters extraction )}

The normal of urodynamic parameters dataset has been obtained from the Institute of Experimental Clinical Research. The dataset consists of eight attributes as shown in the Table 1, which are : maximum detrusor pressure, opening pressure, detrusor pressure at maximum flow rate, volume of urine voided/micturition , maximum urine flow rate , maximum bladder contractility, micturition frequency and number of micturitions .

Table 1: Normal Urodynamic Parameters

\begin{tabular}{cccc}
\hline No & Normal Urodynamic Parameters & Min & Max \\
\hline $\mathbf{1}$ & Max. Detrusor pressure $\left(\mathrm{cm} \mathrm{H}_{2} \mathrm{O}\right)$ & 54.4 & 63.4 \\
$\mathbf{2}$ & Opening pressure $\left(\mathrm{cm} . \mathrm{H}_{2} \mathrm{O}\right)$ & 48 & 54.4 \\
$\mathbf{3}$ & Detrusor pressure at max. flow rate $(\mathrm{cm}$. & 45.6 & 50 \\
& $\left.\mathrm{H}_{2} \mathrm{O}\right)$ & & \\
$\mathbf{4}$ & Vol. Voided $/$ micturition $(\mathrm{ml})$. & 266 & 306 \\
$\mathbf{5}$ & Max. Urine flow rate $(\mathrm{ml} . / \mathrm{sec})$. & 23 & 25.8 \\
$\mathbf{6}$ & Max. Contractility $\left(\mathrm{w} . / \mathrm{m}^{2}{ }^{2}\right)$ & 14 & 16.8 \\
$\mathbf{7}$ & Micturition frequency $(\mathrm{hr})$. & 0.25 & 0.31 \\
$\mathbf{8}$ & No. Micturitions & 5.4 & 6.2 \\
\hline
\end{tabular}

After the normal urodynamic parameters have been identified from the previous table, We can classify as shown in Fig. 2 this data set into two categorical groups, first category is normal values of urodynamic parameters, second category is values over or less than normal value.

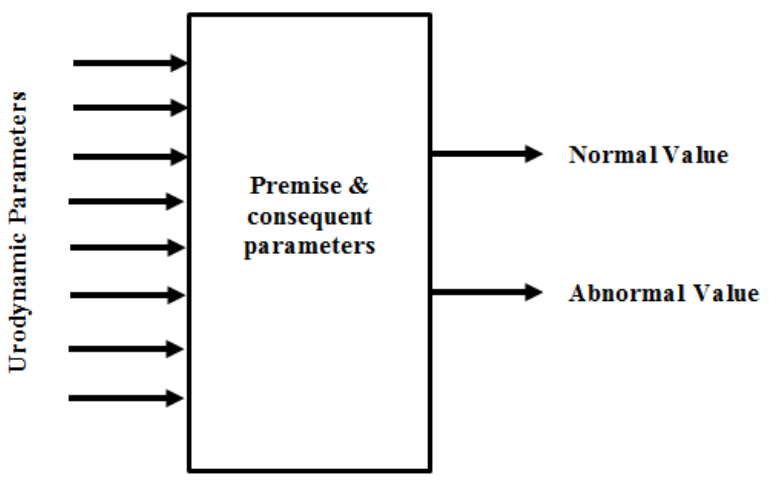

Fig.2: Input/output of ANFIS classifier model

\subsection{Determine the premise and consequent parameters}

In this stage we are training the ANFIS model based on prior urodynamic data to adjust premise and consequent parameters , so that it could take a decision and classify any inputs value into normal or abnormal value The Fuzzy Logic Toolbox of MATLAB provides us a simple way in developing the Adaptive-Network-based Fuzzy Inference System by the ANFIS Editor GUI. The steps required for the ANFIS training can be shown as follows :

\section{Load inputs/outputs data}

In this step we introduce the previous inputs/outputs urodynamic date into ANFIS .The dataset consists of 300 cases divided to 100 normal cases and 200 abnormal cases (over and less than normal urodynamic parameters). Fig.3 shows input/output data set after loading .

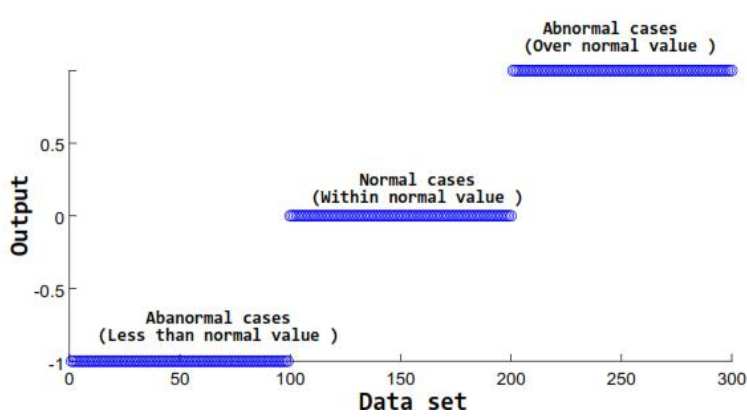

Fig.3: Input/output data set will be used to train a ANFIS

II. Generate an initial FIS structure

We use two Gaussian membership functions as shown in Fig.4 for each input to cover the full range of the respective inputs. Thus, we get 265 rules for the output function which is linear relation of the inputs.
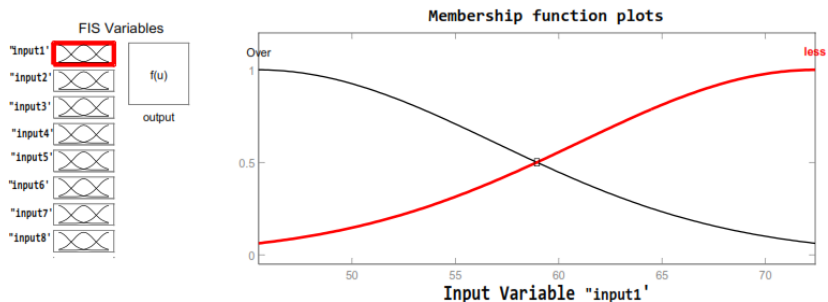

Fig.4: Gaussian membership functions for input 1

III. Select the number of training epochs and the training error tolerance.

The number of training epochs is set 100 and training error tolerance set to 0.02 .

\section{Train the FIS model}

We used a hybrid learning algorithm for the training of the ANFIS network. The ANFIS model achieved Root Mean Squared Error (RMSE) values of 0.02 (error goal) as shown in Fig. 5 and Fig. 6 .

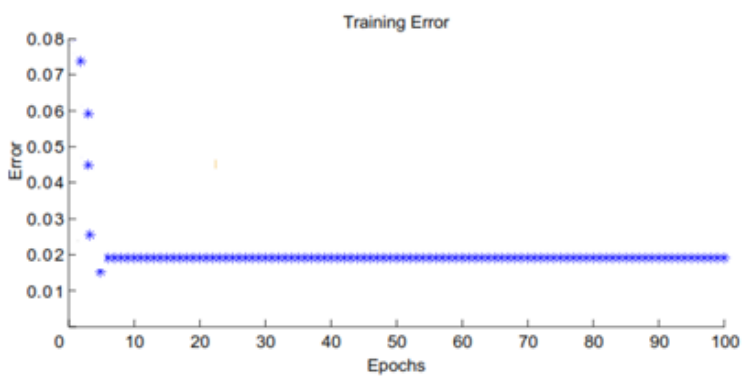

Fig.5: Training error curve 


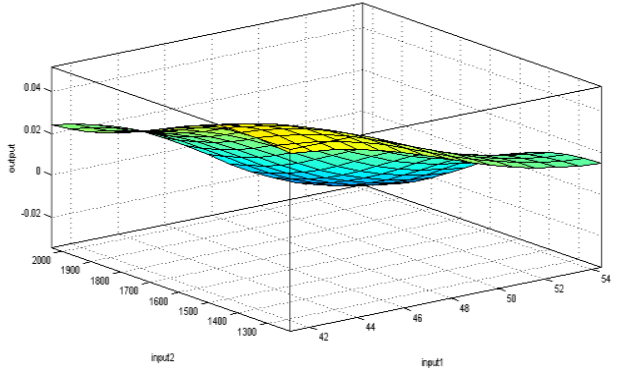

Fig.6: Surface view after ANFIS Training

We note the classification of normal and abnormal cases were done with the accuracy of $97.3 \%$, where ANFIS classifier was able to correctly assign the 292 cases which are satisfying.

\section{SYSTEM VALIDATION AND DISCUSSIONS}

We are generate random 300 case study to test model performance , 100 Case normal , 100 case over normal value and 100 case . To validate the ANFIS classifier we rewriting the equation no (4) after summation by premise and consequent parameters that obtained after ANFIS training and apply this case to ANFIS classifier The results of the model's ability to classification are shown in Table 2.

Table 2: Summary of ANFIS classifier results

\begin{tabular}{cccc}
\hline & $\begin{array}{c}\text { Number } \\
\text { of case }\end{array}$ & $\begin{array}{c}\text { Correctly } \\
\text { decision }\end{array}$ & $\begin{array}{c}\text { Incorrectly } \\
\text { decision }\end{array}$ \\
\hline $\begin{array}{c}\text { Normal } \\
\text { value }\end{array}$ & 100 & 99 & 1 \\
$\begin{array}{c}\text { Over normal } \\
\text { value }\end{array}$ & 100 & 97 & 3 \\
$\begin{array}{c}\text { Less than } \\
\text { normal value }\end{array}$ & 100 & 96 & 4 \\
\hline
\end{tabular}

\section{CONCLUSION}

This thesis suggests a technique for classification of urodynamic testing for rapid decide of the urodynamic test as normal or not value with an accuracy rate of $97.3 \%$ by using Adaptive Neuro-Fuzzy Inference System (ANFIS) as classification system. In the future an attempt will add a new complex inputs parameters for ANFIS classifier model .

\section{ACKNOWLEDGEMENTS}

The authors would like to gratefully acknowledge Prof. Ahmed M. El-Garhy and Assoc.Prof.Dr Ahmed S.El-Azab, for their helpful comments in preparation of this article

\section{REFERENCES}

[1] F. Schmidt, P. Shin, T. M. Jorgensen, J. C. Djurhuus, and C. E. Constantinou, "Urodynamic patterns of normal male micturition: influence of water consumption on urine production and detrusor function," J. Urol., vol. 168, pp. 1458-1463, 2002.

[2] G. A. Van Koeveringe, B. Vahabi, P. H. D. K. E. Andersson, and P. H. D. R. Kirschner-herrmans, "Detrusor Underactivity: A Plea for New Approaches to a Common Bladder Dysfunction," Neurourol. Urodyn., vol. 728, no. June, pp. 723-728, 2011.
[3] RA. Hosein and D.J. Griffiths. Computer simulation of the neural control of bladder and urethra. Neurourology and Urodynamics, 9:601 618, 1990.

[4] G. Rutishauser and P. Graber. Normal micturition. In W. Lutzeyer and $\mathrm{H}$. Melchoir, editors, Urodynamics, upper and lower urinary tract., pages 199-203. Springer-Verlag, Berlin, Heidelberg, New York, 1973.

[5] "Urodynamic Patterns of Normal Male Micturition: Influence of Water Consumption on Urine Production and Detrusor Function", Schmidt, Shin, Jorgensen, Djurhuus and Constantinou J. Urol. 168: 1458-1463 (2002)

[6] "Urodynamic Model of the Lower Urinary Tract", Pay, Chamizo, Pic and Pérez Concurrent Systems Engineering Series 54: 123-128 (1999)

[7] "A Mathematical Micturition Model to Restore Simple Flow Recordings in Healthy and Symptomatic Individuals and Enhance Uroflow Interpretation", Valentini, Besson, Nelson and Zimmern Neurourol. Urodyn. 19: 153-176 (2000)

[8] "Modelized Analysis of Pressure-Flow studies of Patients With Lower Urinary Tract Symptoms Due to Benign Prostatic Enlargement", Valentini, Zimmern, Besson and Nelson Neurourol. Urodyn. 22: 45-53 2003

[9] "Finite Element Models - A Template for Future Urodynamics: Comparison of Different Computer Models of the Neural Control System of the Lower Urinary Tract", Petros Neurourol. Urodynam. 19: 289 $310(2000)$

[10] Opeyemi, O., Justice, E. O., Development of Neurofuzzy System for Early Prediction of Heart Attack, Information Technology and Computer Science, Vol:9, pp. 22-28, 2012.

[11] Askerbeyli.I \&Aybike, F., Effects of fuzzy logic methods over ATM networks, International Journal of Research and Reviews in Applied Sciences, Vol.9, pp. 295-305, 2011.

[12] W. Fletcher, F. T. Smith, and C. Fry, "A Computer Simulation of Micturition," London, 2005.

[13] Y. Z. Jafri, A. Waseem, L. Kamal, S. M. Raza, and M. Sami, "Chaotic time series prediction and Mackey-Glass simulation with fuzzy logic," Int. J. Phys. Sci., vol. 7, no. 17, pp. 2596-2606, Apr. 2012.

[14] Allouche Moez, Souissi Mansour, Chaabane Mohamed and Mehdi Driss, "Takagi-Sugeno Fuzzy Control of Induction Motor", Proc. Int. Journal of Electrical and Electronics Engg., Vol. 2, Issue 1, 2009.

[15] F.-y. Su, Z. Wen, "Application of fuzzy control technology in temperature setting of regenerative rotary reheating furnace", "IEEE/FSKD", Vol.3, pp.1205-1208, Aug. 2010.

[16] D. Su, K. Ren, J. Luo, C. He, L. Wang, X. Zhang, "Programmed and simulation of the fuzzy control list in fuzzy control", "IEEE/WCICA", pp.1935-1940, July 2010.

[17] H. Chung, J. Lee, S. Joe, D. Chung, S. Kim, M. Chung, Application of fuzzy PID controller for stabilization of power system", "IEEE/FUZZY", Vol.3, pp.1729-1733, Seoul, 1999. 